\title{
Dynamics of a Nonautonomous SIR Model with Time-Varying Impulsive Release and General Nonlinear Incidence Rate in a Polluted Environment
}

\author{
Fumin Zhang, Shujing Gao, Yujiang Liu, Yan Zhang \\ Key Laboratory of Jiangxi Province for Numerical Simulation and Emulation Techniques, Gannan Normal \\ University, Ganzhou, China \\ Email: gaosjmath@126.com
}

Received 7 March 2016; accepted 25 April 2016; published 28 April 2016

Copyright (C) 2016 by authors and Scientific Research Publishing Inc.

This work is licensed under the Creative Commons Attribution International License (CC BY).

http://creativecommons.org/licenses/by/4.0/

(c) (i) Open Access

\begin{abstract}
In a polluted environment, considering the biological population infected with a kind of disease and hunted by human beings, we formulate a nonautonomous SIR population-epidemic model with time-varying impulsive release and general nonlinear incidence rate and investigate dynamical behaviors of the model. Under the reasonable assumptions, the sufficient conditions which guarantee the globally attractive of the disease-free periodic solution and the permanence of the infected fish are established, that is, the infected fish dies out if $R^{*}<1$, whereas the disease persists if $R_{*}>1$. To substantiate our theoretical results, extensive numerical simulations are performed for a hypothetical set of parameter values.
\end{abstract}

\section{Keywords}

Nonautonomous SIR Model, Varying Pulses, General Nonlinear Incidence Rate, Global Attractivity

\section{Introduction}

It is well known that Poyang Lake located in the middle and lower reaches of the Yangtze River is the current largest freshwater lake in China. Its wetland ecosystem has a significant impact on the change of China's environment. The sufficient water resource and the superior natural environment nurture the abundant aquatic living resources of Poyang Lake. There are 136 kinds of fishes, 87 kinds of shells, 102 kinds of aquatic vascular 
plants and 266 kinds of identified plankton in Poyang Lake. The fishes in Poyang Lake take up 16.39\% of the fresh water fish varieties in China, and $36.76 \%$ of the fish varieties of Yangtze River system. There are also first-level and second-level national protected precious rare aquatic animals such as white-flag dolphin, cowfish, chinese sturgeon, hilsa herring and so on in Poyang Lake, making it known as the treasury of fishery resources and the fish species genetic base with a significant position in the ecology system of the fish industry of Yangtze River reaches [1].

At present, the grand development of Poyang Lake ecological economy is under way in a large scale in province, which promotes the establishment of the ecological economy zone [2]. However, the rapid economic development of Poyang Lake will have a negative influence on the living circumstances of fishes in the area. For the past few years, with the rapid development of modern industry and agriculture, a great quantity of toxicant and contaminants enter into Poyang Lake wetland ecosystem one after another. In order to use and regulate toxic substances wisely, we must assess the risk of the populations exposed to toxicant. Therefore, it is very important to investigate the effects of toxicants on populations and to find a theoretical threshold value, which determines permanence or extinction of fish population or community.

In recent years, many scholars have been conducted to investigate the effect of toxicant emitted into the environment from industrial, agricultural and household sources on biological species [3]-[19] by using mathematical models. For instance, Wang and Ma [18] investigated a nonautonomous SIS epidemic model with toxicant influence. They showed the existence and global attractiveness of periodic solutions and obtained the threshold between extinction and weak persistence of the infected class. Liu and Duan [19] considering the biological population infected with some kinds of diseases and hunted by human beings, and they formulate two SI pollution-epidemic models with continuous and impulsive external effects, respectively, and investigate the dynamics of such systems. But these previous models have invariably assumed that the exogenous input of toxicant is continuous or emitted in regular pulses. However, in the real life, it is often the case that toxicant is emitted in irregular pulses. In this paper, according to the above biological background, we investigate a nonautonomous SIR population-epidemic model with time-varying impulsive release and general nonlinear incidence rate and study dynamical behaviors of the model.

The organization of this paper is as follows. In the next section, we give some useful notations, definitions and preliminary lemmas which will be used to proof our main results. In Section 3, we mainly investigate a nonautonomous mathematical model with general nonlinear incidence rate and time-varying impulsive release, under some assumptions and the biological interpretation. In Section 4, we show that global attractivity of the disease-free periodic solution is determined by the threshold parameter $R^{*}$. In Section 5 , we give another expression of threshold parameter $R_{*}$, and show that if $R_{*}>1$, the disease is permanent. In the last section, we give a brief discussion and some numerical simulation results which conform the theoretical conclusions.

\section{Notations, Definitions and Preliminary Lemmas}

In this section, we introduce some notations, definitions and state some lemmas which will be useful in the subsequent sections. Let $C$ denote the space of all bounded continuous functions. Given $f \in C$, we let

$$
f^{M}=\sup _{t \geq 0} f(t), \quad f^{L}=\inf _{t \geq 0} f(t) .
$$

If $f$ is $\omega$-periodic, then the average value of $f$ on a time interval $[0, \omega]$ can be defined as

$$
\bar{f}=\frac{1}{\omega} \int_{0}^{\omega} f(t) \mathrm{d} t .
$$

Before demonstrating the global attractivity of disease-free periodic solution of system (7), we need to introduce an important lemma.

Lemma 1. (see [20]) Consider the following nonautonomous linear differential equation:

$$
\frac{\mathrm{d} x(t)}{\mathrm{d} t}=a(t)-b(t) x(t)
$$

where $a(t)$ and $b(t)$ are continuous and positive $\omega$-periodic functions. Then the system has a unique positive $\omega$-periodic solution $x^{*}(t)$ which is globally asymptotically stable. 


\section{Model Formulation and Preliminary}

First of all, the total freshwater fish is divided into three groups: Susceptible fish $(S)$, Infected fish $(I)$ and Removed fish $(R)$. Motivated by the above works and these literatures [21]-[29], now we investigate the properties of fish's dynamical behaviour of the model and human intervention in the polluted environment. The system is modeled by the following equations:

$$
\left\{\begin{array}{l}
\frac{\mathrm{d} S(t)}{\mathrm{d} t}=\Lambda(t)-f(t, S, I)-d_{1}(t) S(t)-\alpha_{1}(t) C_{0}(t) S(t), \\
\frac{\mathrm{d} I(t)}{\mathrm{d} t}=f(t, S, I)-d_{2}(t) I(t)-\alpha_{2}(t) C_{0}(t) I(t)-\mu(t) I(t), \\
\frac{\mathrm{d} R(t)}{\mathrm{d} t}=\mu(t) I(t)-d_{3}(t) R(t)-\alpha_{3}(t) C_{0}(t) R(t), \\
\frac{\mathrm{d} C_{0}(t)}{\mathrm{d} t}=k(t) C_{E}(t)-g(t) C_{0}(t)-m(t) C_{0}(t), \\
\frac{\mathrm{d} C_{E}(t)}{\mathrm{d} t}=-h(t) C_{E}(t), \\
S\left(t_{k}^{+}\right)=S\left(t_{k}\right), \\
I\left(t_{k}^{+}\right)=I\left(t_{k}\right), \\
R\left(t_{k}^{+}\right)=R\left(t_{k}\right), \\
C_{0}\left(t_{k}^{+}\right)=C_{0}\left(t_{k}\right), \\
C_{E}\left(t_{k}^{+}\right)=C_{E}\left(t_{k}\right)+p_{k},
\end{array}\right\} t \neq t_{k}, k \in \mathbb{N}
$$

The model is derived with the following assumptions.

- $S(t), I(t)$ and $R(t)$ represent the density of susceptible fish, infected fish and removed fish at time $t$, respectively. The initial conditions are $S\left(t_{0}\right)>0, I\left(t_{0}\right)>0$ and $R\left(t_{0}\right)>0$.

- $S(t), I(t)$ and $R(t)$ are left continuous for $\left[t_{0},+\infty\right)$, that is, $S(t)=\lim _{h \rightarrow 0^{+}} S(t-h)$, $I(t)=\lim _{h \rightarrow 0^{+}} I(t-h)$ and $R(t)=\lim _{h \rightarrow 0^{+}} R(t-h)$.

- $\Lambda(t), d_{i}(t)(i=1,2,3)$ are the instantaneous recruitment rate, death rate at time $t$, respectively. $\alpha_{i}(t)$ $(i=1,2,3)$ is the dose response parameter of the susceptible, infected and removed populations.

- $C_{0}(t)$ and $C_{E}(t)$ represent the concentration of population in the organism and in the environment at time $t$, respectively. $k C_{E}(t)$ represents the organisms net uptake of population from the environment. $-g(t) C_{0}(t)$ and $-m(t) C_{0}(t)$ represent the egestion and depuration rates of population int the organism, respectively. $-h(t) C_{E}(t)$ represents the loss of population in the environment due to natural degradation.

- The coefficients $\Lambda(t), \mu(t), k(t), g(t), m(t), h(t), \alpha_{i}(t)$ and $d_{i}(t)(i=1,2,3)$ are assumed to be nonnegative, continuous and bounded $\omega$-periodic functions in the interval $\left[t_{0},+\infty\right)$.

- There exists a positive integer $q$ such that $t_{k+q}=t_{k}+\omega$ for all $k \in \mathbb{N}$. The exogenous quantity of impulsive input of toxin into the environment is represented by $p_{k}$ at each fix time, and $p_{k}=p_{q+k}$ for $k \in \mathbb{N}$.

- The general nonlinear incidence rate $f(t, S, I)$ is a piecewise continuous, nonnegative, periodic function with period $\omega$. The form of $f(t, S, I)$ is as follows:

$$
f(t, S, I)=\left\{\begin{array}{cl}
f_{1}(t, S, I), & t \in\left(n \omega+t_{0}, n \omega+t_{1}\right], \\
\vdots & \\
f_{q}(t, S, I), & t \in\left(n \omega+t_{q-1}, n \omega+t_{q}\right],
\end{array}\right.
$$

for all integer $n \geq 0$, and $f_{i}(t, 0, I)=f_{i}(t, S, 0)=0$, for $i=1,2, \cdots, q$. 
In the following, we give some basic properties of the following subsystem of model (1), which are very important for deriving our main results.

$$
\left\{\begin{array}{l}
\frac{\mathrm{d} C_{0}(t)}{\mathrm{d} t}=k(t) C_{E}(t)-g(t) C_{0}(t)-m(t) C_{0}(t), \\
\frac{\mathrm{d} C_{E}(t)}{\mathrm{d} t}=-h(t) C_{E}(t), \\
C_{E}\left(t_{k}^{+}\right)=C_{E}\left(t_{k}\right)+p_{k}, \\
C_{0}\left(t_{0}^{+}\right)=C_{0}\left(t_{0}\right),
\end{array}\right\} t=t_{k}, k \in \mathbb{N} .
$$

where $0 \leq C_{0}\left(t_{0}\right) \leq 1, \quad 0 \leq C_{E}\left(t_{0}\right) \leq 1$.

Lemma 2. System (2) has a unique positive $\omega$-periodic solution $\left(C_{0}^{*}(t), C_{E}^{*}(t)\right)^{\mathrm{T}}$ which is globally asymptotically stable, where

$$
\begin{gathered}
C_{E}^{*}(t)=\left[C_{E 0}^{*} \exp \left(-\int_{n \omega+t_{0}}^{n \omega+t_{k}} h(s) \mathrm{d} s\right)+\sum_{k=1}^{q} \exp \left(-\int_{n \omega+t_{k}}^{n \omega+t_{q}} h(s) \mathrm{d} s\right)\right] \times \exp \left(-\int_{n \omega+t_{k-1}}^{t} h(s) \mathrm{d} s\right), \\
C_{E 0}^{*}=\frac{\sum_{k=1}^{q} p_{k} \exp \left(-\int_{n \omega+t_{k}}^{n \omega+t_{q}} h(s) \mathrm{d} s\right)}{1-\exp \left(-\int_{n \omega+t_{0}}^{n \omega+t_{q}} h(s) \mathrm{d} s\right)} .
\end{gathered}
$$

for $n \omega+t_{k-1}<t \leq n \omega+t_{k}, \quad k=1,2, \cdots, q, \quad k \in \mathbb{N}$.

Proof. Integrating and solving the first equation of system (2) between pulses for $n \omega+t_{k-1}<t \leq n \omega+t_{k}$, $k=1,2, \cdots, q, \quad k \in \mathbb{N}$,

where

$$
C_{E}(t)=C_{E}\left(\left(n \omega+t_{k-1}\right)^{+}\right) \exp \left(-\int_{n \omega+t_{k-1}}^{t} h(s) \mathrm{d} s\right)
$$

$$
C_{E}\left(\left(n \omega+t_{k-1}\right)^{+}\right)=C_{E}\left(n \omega+t_{k-1}\right)+p_{k},
$$

and $C_{E}\left(n \omega+t_{k-1}\right)$ be the initial value at time $n \omega+t_{k-1}$.

It follows from above equation and using the third equation of system (2), we get

and

$$
C_{E}\left(\left(n \omega+t_{1}\right)^{+}\right)=C_{E}\left(\left(n \omega+t_{0}\right)^{+}\right) \exp \left(-\int_{n \omega+t_{0}}^{n \omega+t_{1}} h(s) \mathrm{d} s\right)+p_{1},
$$

$$
C_{E}\left(\left(n \omega+t_{2}\right)^{+}\right)=C_{E}\left(\left(n \omega+t_{0}\right)^{+}\right) \exp \left(-\int_{n \omega+t_{0}}^{n \omega+t_{2}} h(s) \mathrm{d} s\right)+p_{1} \exp \left(-\int_{n \omega+t_{1}}^{n \omega+t_{2}} h(s) \mathrm{d} s\right)+p_{2} .
$$

Obviously, $C_{E}\left(\left(n \omega+t_{q}\right)^{+}\right)=C_{E}\left(\left((n+1) \omega+t_{0}\right)^{+}\right)$, using the inductive method, we have

$$
C_{E}\left(\left(n \omega+t_{q}\right)^{+}\right)=C_{E}\left(\left(n \omega+t_{0}\right)^{+}\right) \exp \left(-\int_{n \omega+t_{0}}^{n \omega+t_{q}} h(s) \mathrm{d} s\right)+\sum_{k=1}^{q} p_{k} \exp \left(-\int_{n \omega+t_{k}}^{n \omega+t_{q}} h(s) \mathrm{d} s\right) .
$$

Set $U_{n}=C_{E}\left(n \omega+t_{0}\right)$. From (5) and $t_{q}-t_{0}=\omega$, we have

$$
U_{n+1}=U_{n} \exp \left(-\int_{n \omega+t_{0}}^{n \omega+t_{q}} h(s) \mathrm{d} s\right)+\sum_{k=1}^{q} p_{k} \exp \left(-\int_{n \omega+t_{k}}^{n \omega+t_{q}} h(s) \mathrm{d} s\right) \triangleq f\left(U_{n}\right) .
$$

$f$ is the stroboscopic map. It is easy to see that system (6) has a unique positive equilibrium:

$$
C_{E 0}^{*}=\frac{\sum_{k=1}^{q} p_{k} \exp \left(-\int_{n \omega+t_{k}}^{n \omega+t_{q}} h(s) \mathrm{d} s\right)}{1-\exp \left(-\int_{n \omega+t_{0}}^{n \omega+t_{q}} h(s) \mathrm{d} s\right)} .
$$


Since $f\left(U_{n}\right)$ is a straight line with slope less than 1 , we obtain that $C_{E 0}^{*}$ is globally asymptotically stable. It implies that the corresponding periodic solution of system (2) $C_{E}^{*}(t)$ is globally asymptotically stable. Furthermore, according to Lemma 1 , we can obtain that the system (2) has a unique positive $\omega$-periodic solution $\left(C_{0}^{*}(t), C_{E}^{*}(t)\right)$ which is globally asymptotically stable. Therefore, the limit system of (1) is as follows:

$$
\left\{\begin{array}{l}
\frac{\mathrm{d} S(t)}{\mathrm{d} t}=\Lambda(t)-f(t, S, I)-d_{1}(t) S(t)-\alpha_{1}(t) C_{0}^{*}(t) S(t), \\
\frac{\mathrm{d} I(t)}{\mathrm{d} t}=f(t, S, I)-d_{2}(t) I(t)-\alpha_{2}(t) C_{0}^{*}(t) I(t)-\mu(t) I(t), \\
\frac{\mathrm{d} R(t)}{\mathrm{d} t}=\mu(t) I(t)-d_{3}(t) R(t)-\alpha_{3}(t) C_{0}^{*}(t) R(t) .
\end{array}\right.
$$

By Lemma 1, it is easy to see that system (7) has a unique disease-free periodic solution $\left(S^{*}(t), 0,0\right)$.

\section{Global Attractivity of the Disease-Free Periodic Solution}

To discuss the attractivity of the disease-free periodic solution of system (7), we firstly give the following hypothesis:

(A) There exist positive, continuous, periodic functions $\beta_{k}(t)$ with period $\omega$, such that $f_{i}(t, S, I) \leq$ $\beta_{i}(t) S(t) I(t)$, for all $i=1,2, \cdots, q$, and $t \geq t_{0}$.

Theorem 1. If $R^{*}<1$ and system (7) satisfies the Hypothesis (A), then the disease-free solution $\left(S^{*}(t), 0,0\right)$ is globally attractive, where

$$
R^{*}=\frac{\sum_{i=1}^{q} \int_{t_{i-1}}^{t_{i}} \beta_{i}(\tau) S^{*}(\tau) \mathrm{d} \tau}{\sum_{i=1}^{q} \int_{t_{i-1}}^{t_{i}}\left[d_{2}(\tau)+\alpha_{2}(\tau) C_{0}^{*}(\tau)+\mu(\tau)\right] \mathrm{d} \tau} .
$$

Proof. Let $(S(t), I(t), R(t))$ be any solution of system (7). Since $R^{*}<1$, we can choose a sufficiently small number $\varepsilon_{1}>0$ such that

$$
\Omega_{1} \triangleq \exp \left[\sum_{i=1}^{q} \int_{t_{i-1}}^{t_{i}} \beta_{i}(\tau)\left(S^{*}(\tau)+\varepsilon_{1}\right) \mathrm{d} \tau-\sum_{i=1}^{q} \int_{t_{i-1}}^{t_{i}}\left[d_{2}(\tau)+\alpha_{2}(\tau) C_{0}^{*}(\tau)+\mu(\tau)\right] \mathrm{d} \tau\right]<1 .
$$

From the second equation of system (7), we obtain that

$$
\frac{\mathrm{d} S(t)}{\mathrm{d} t} \leq \Lambda(t)-d_{1}(t) S(t)-\alpha_{1}(t) C_{0}^{*}(t) S(t) .
$$

By the comparison theorem, we can get that there exists a constant $t^{1}\left(>t_{0}\right)$ such that

$$
S(t)<S^{*}(t)+\varepsilon_{1}
$$

for all $t \geq t^{1}$.

It follows from (9) and the second equation of system (7) that, for

$$
\begin{aligned}
& t \in\left(n \omega+t_{i-1}, n \omega+t_{i}\right](i=1,2, \cdots, q) \text { and } t \geq t^{1}, \\
\frac{\mathrm{d} I(t)}{\mathrm{d} t}= & f_{i}(t, S, I)-d_{2}(t) I(t)-\alpha_{2}(t) C_{0}^{*}(t) I(t)-\mu(t) I(t) \\
\leq & \beta_{i}(t) S(t) I(t)-d_{2}(t) I(t)-\alpha_{2}(t) C_{0}^{*}(t) I(t)-\mu(t) I(t) \\
\leq & {\left[\beta_{i}(t)\left(S^{*}(t)+\varepsilon_{1}\right)-\left(d_{2}(t)+\alpha_{2}(t) C_{0}^{*}(t)+\mu(t)\right)\right] I(t) . }
\end{aligned}
$$

Then, we obtain that

$$
I(t) \leq I\left(n \omega+t_{i-1}\right) \exp \left[\int_{n \omega+t_{i-1}}^{t}\left[\beta_{i}(\tau)\left(S^{*}(\tau)+\varepsilon_{1}\right)-\left(d_{2}(\tau)+\alpha_{2}(t) C_{0}^{*}(\tau)+\mu(\tau)\right)\right] \mathrm{d} \tau\right] .
$$


By using the similar method, we can infer that for $t \in\left(n \omega+t_{i-1}, n \omega+t_{i}\right]$

$$
\begin{aligned}
I(t) \leq & I\left(n \omega+t_{0}\right) \times \exp \left[\int_{n \omega+t_{0}}^{n \omega+t_{1}} \beta_{1}(\tau)\left(S^{*}(\tau)+\varepsilon_{1}\right) \mathrm{d} \tau+\cdots+\int_{n \omega+t_{i-1}}^{t} \beta_{i}(\tau) \mathrm{d} \tau\left(S^{*}(\tau)+\varepsilon_{1}\right) \mathrm{d} \tau\right] \\
& \times \exp \left[\int_{n \omega+t_{0}}^{n \omega+t_{1}}\left[-\left(d_{2}(\tau)+\alpha_{2}(\tau) C_{0}^{*}(\tau)+\mu(\tau)\right)\right] \mathrm{d} \tau\right] \times \cdots \\
& \times \exp \left[\int_{n \omega+t_{i-1}}^{t}\left[-\left(d_{2}(\tau)+\alpha_{2}(\tau) C_{0}^{*}(\tau)+\mu(\tau)\right)\right] \mathrm{d} \tau\right] \\
= & I\left(n \omega+t_{0}\right) \exp \left[\sum_{j=1}^{i} \int_{n \omega+t_{j-1}}^{n \omega+t_{j}} \beta_{i}(\tau)\left(S^{*}(\tau)+\varepsilon_{1}\right) \mathrm{d} \tau\right] \\
& \times \exp \left[\sum_{j=1}^{i} \int_{n \omega+t_{j-1}}^{n \omega+t_{j}}\left[-\left(d_{2}(\tau)+\alpha_{2}(\tau) C_{0}^{*}(\tau)+\mu(\tau)\right)\right] \mathrm{d} \tau\right] .
\end{aligned}
$$

Especially, when $t=(n+1) \omega+t_{0}$, we have

$$
\begin{aligned}
I\left((n+1) \omega+t_{0}\right)= & I\left(n \omega+t_{q}\right) \\
\leq & I\left(n \omega+t_{0}\right) \exp \left[\sum_{j=1}^{q} \int_{t_{j-1}}^{t_{j}} \beta_{i}(\tau)\left(S^{*}(t)+\varepsilon_{1}\right) \mathrm{d} \tau\right] \\
& \times \exp \left[\sum_{j=1}^{q} \int_{t_{j-1}}^{t_{j}}\left[-\left(d_{2}(\tau)+\alpha_{2}(\tau) C_{0}^{*}(\tau)+\mu(\tau)\right)\right] \mathrm{d} \tau\right] \\
& =\Omega_{1} I\left(n \omega+t_{0}\right) .
\end{aligned}
$$

Therefore, we have $I\left(\left(n+l_{1}\right) \omega+t_{0}\right) \leq \Omega_{1}^{l_{1}} I\left(n \omega+t_{0}\right)$ for any positive integer $l_{1}$. It follows from (9) that

$$
I\left(\left(n+l_{1}\right) \omega+t_{0}\right) \rightarrow 0 \text {, as } l_{1} \rightarrow+\infty .
$$

From the (10) and (11), we get

$$
\lim _{t \rightarrow+\infty} I(t)=0 .
$$

Therefore, for above mentioned $\varepsilon_{1}$, there exist $t^{2}\left(>t^{1}\right)$, we have

$$
I(t)<\varepsilon_{1},
$$

for all $t>t^{2}$. From the first and third equation of system (6) and (12), we have for $t>t^{2}$,

$$
\begin{aligned}
\frac{\mathrm{d} R(t)}{\mathrm{d} t} & =\mu(t) I(t)-d_{3}(t) R(t)-\alpha_{3}(t) C_{0}(t) R(t) \\
& \geq-\left(d_{3}(t) R(t)+\alpha_{3}(t) C_{0}^{*}(t)\right) R(t),
\end{aligned}
$$

and

$$
\begin{aligned}
\frac{\mathrm{d} R(t)}{\mathrm{d} t} & =\mu(t) I(t)-d_{3}(t) R(t)-\alpha_{3}(t) C_{0}(t) R(t) \\
& \leq \mu(t) \varepsilon_{1}-\left(d_{3}(t) R(t)+\alpha_{3}(t) C_{0}^{*}(t)\right) R(t),
\end{aligned}
$$

where $\varepsilon_{1}$ is a sufficiently small number. Thus, we get

$$
\lim _{t \rightarrow+\infty} R(t)=0 .
$$

By using the similar method, we can see that

$$
\begin{aligned}
\frac{\mathrm{d} S(t)}{\mathrm{d} t} & =\Lambda(t)-f_{k}(t, S, I)-d_{1}(t) S(t)-\alpha_{1}(t) C_{0}(t) S(t) \\
& \leq \Lambda(t)-\left(d_{1}(t) S(t)+\alpha_{1}(t) C_{0}^{*}(t)\right) S(t)
\end{aligned}
$$


and

$$
\begin{aligned}
\frac{\mathrm{d} S(t)}{\mathrm{d} t} & =\Lambda(t)-f_{k}(t, S, I)-d_{1}(t) S(t)-\alpha_{1}(t) C_{0}(t) S(t) \\
& \geq \Lambda(t)-\left(\beta_{k}(t) \varepsilon_{1}+d_{1}(t) S(t)+\alpha_{1}(t) C_{0}^{*}(t)\right) S(t),
\end{aligned}
$$

where $\varepsilon_{1}$ is an arbitrary small. Therefore, we also obtain that

$$
\lim _{t \rightarrow+\infty}\left(S(t)-S^{*}(t)\right)=0
$$

From (14) and (15), we can see that the disease-free periodic solution $\left(S^{*}(t), 0,0\right)$ is global attractive.

\section{Permanence of the Disease}

In this section, we mainly obtain the sufficient conditions for the permanence of system (7). Therefore, we give the following hypotheses at first.

(B) There exist positive, continuous, periodic functions $\gamma_{i}(t)$ with the periodic $\omega$, such that $f_{i}(t, S, I) \geq$ $\gamma_{i}(t) S(t) I(t)$, for $i=1,2, \cdots, q$ and $t \geq t_{0}$. Denote $S^{* *}(t)$ be the solution of the following system:

$$
\frac{\mathrm{d} Z(t)}{\mathrm{d} t}=\Lambda(t)-\left(\beta_{k}(t) \eta+d_{1}(t)+\alpha_{1}(t) C_{0}^{*}(t)\right) Z(t) .
$$

According to Lemma 1, we can obtain that the system has a unique positive $\omega$-periodic solution $S^{* * *}(t)$ which is globally asymptotically stable.

Theorem 2. If $R_{*}>1$ and system (7) satisfies the Hypotheses (A) and (B), then system (7) is permanent, where

$$
R_{*}=\frac{\sum_{i=1}^{q} \int_{t_{i-1}}^{t_{i}} \gamma_{i}(\tau) S^{* *}(\tau) \mathrm{d} \tau}{\sum_{i=1}^{q} \int_{t_{i-1}}^{t_{i}}\left[d_{2}(\tau)+\alpha_{2}(\tau) C_{0}^{*}(\tau)+\mu(\tau)\right] \mathrm{d} \tau} .
$$

Proof. Since $R_{*}>1$, we can easily see that there exists a sufficiently small $\eta>0$ such that

$$
\Omega_{2} \triangleq \exp \left[\sum_{i=1}^{q} \int_{t_{i-1}}^{t_{i}} \gamma_{i}(\tau)\left(S^{* *}(\tau)-\eta\right) \mathrm{d} \tau-\sum_{i=1}^{q} \int_{t_{i-1}}^{t_{i}}\left[d_{2}(\tau)+\alpha_{2}(\tau) C_{0}^{*}(\tau)+\mu(\tau)\right] \mathrm{d} \tau\right]>1 .
$$

In order to illustrate the conclusion, we firstly obtain the disease is uniformly weakly persistent, that is, there exists a positive constant $\sigma>0$, such that $\limsup I(t) \geq \sigma$. By contradiction, we have that, for all given $\eta>0$, there exists a $t^{3}>0$ such that $I(t)<\eta$ for all $t>t^{3}$.

In view of the Hypothesis (A) and the first equation of system (7), we get

$$
\begin{aligned}
\frac{\mathrm{d} S(t)}{\mathrm{d} t} & =\Lambda(t)-f_{k}(t, S, I)-d_{1}(t) S(t)-\alpha_{1}(t) C_{0}^{*}(t) S(t) \\
& \geq \Lambda(t)-\left(\beta_{k}(t) I(t)+d_{1}(t)+\alpha_{1}(t) C_{0}^{*}(t)\right) S(t) \\
& \geq \Lambda(t)-\left(\beta_{k}(t) \eta+d_{1}(t)+\alpha_{1}(t) C_{0}^{*}(t)\right) S(t) .
\end{aligned}
$$

By comparison theorem, we have $S(t) \geq Z(t)$ and $Z(t) \rightarrow S^{* *}(t)$ as $t \rightarrow+\infty$, where $Z(t)$ is the solution of the following comparison system:

$$
\frac{\mathrm{d} Z(t)}{\mathrm{d} t}=\Lambda(t)-\left(\beta_{k}(t) \eta+d_{1}(t)+\alpha_{1}(t) C_{0}^{*}(t)\right) Z(t)
$$

Therefore, for above mentioned $\eta$, there exists a $n^{*}>0$, such that

$$
S(t) \geq Z(t) \geq S^{* *}(t)-\eta
$$

for all $t>n^{*} \omega+t^{3}$.

For above mentioned $n^{*} \omega+t^{3}$, we have know that there exists a positive integer $n_{1}$ such that $n_{1} \omega>n^{*} \omega+t^{3}$. 
Then for all $n \omega+t_{i-1}<t<n \omega+t_{i} \quad\left(n \geq n_{1}, i=1,2, \cdots, q\right)$, by (17) and the second equation of system (6), we have

$$
\begin{aligned}
\frac{\mathrm{d} I(t)}{\mathrm{d} t} & =f_{i}(t, S, I)-d_{2}(t) I(t)-\alpha_{2}(t) C_{0}^{*}(t) I(t)-\mu(t) I(t) \\
& \geq \gamma_{i}(t) S(t) I(t)-d_{2}(t) I(t)-\alpha_{2}(t) C_{0}^{*}(t) I(t)-\mu(t) I(t) \\
& \geq\left[\gamma_{i}(t)\left(S^{* *}(t)-\eta\right)-\left(d_{2}(t)+\alpha_{2}(t) C_{0}^{*}(t)+\mu(t)\right)\right] I(t) .
\end{aligned}
$$

Then we obtain that

$$
\begin{aligned}
I(t) \geq & I\left(n \omega+t_{0}\right) \times \exp \left[\int_{n \omega+t_{i-1}}^{t} \gamma_{i}(\tau)\left(S^{* *}(\tau)-\eta\right) \mathrm{d} \tau\right] \\
& \times \exp \left[\int_{n \omega+t_{i-1}}^{t}\left[-\left(d_{2}(\tau)+\alpha_{2}(\tau) C_{0}^{*}(\tau)+\mu(\tau)\right)\right] \mathrm{d} \tau\right] .
\end{aligned}
$$

By using the similar method, we can get that for $t \in\left(n \omega+t_{i-1}, n \omega+t_{i}\right]$

$$
\begin{aligned}
I(t) \geq & I\left(n \omega+t_{0}\right) \times \exp \left[\int_{n \omega+t_{0}}^{n \omega+t_{1}} \gamma_{1}(\tau)\left(S^{* * *}(\tau)-\eta\right) \mathrm{d} \tau+\cdots+\int_{n \omega+t_{i-1}}^{t} \gamma_{i}(\tau)\left(S^{* * *}(\tau)-\eta\right) \mathrm{d} \tau\right] \\
& \times \exp \left[\int_{n \omega+t_{0}}^{n \omega+t_{1}}\left[-\left(d_{2}(\tau)+\alpha_{2}(\tau) C_{0}^{*}(\tau)+\mu(\tau)\right)\right] \mathrm{d} \tau\right] \times \cdots \\
& \times \exp \left[\int_{n \omega+t_{i-1}}^{t}\left[-\left(d_{2}(\tau)+\alpha_{2}(\tau) C_{0}^{*}(\tau)+\mu(\tau)\right)\right] \mathrm{d} \tau\right] .
\end{aligned}
$$

Furthermore, when $t=(n+1) \omega+t_{0}$, we have

$$
\begin{aligned}
I\left((n+1) \omega+t_{0}\right)= & I\left(n \omega+t_{q}\right) \\
\geq & I\left(n \omega+t_{0}\right) \exp \left[\sum_{i=1}^{q} \int_{t_{i-1}}^{t_{i}} \gamma_{i}(\tau)\left(S^{* *}(\tau)-\eta\right) \mathrm{d} \tau\right] \\
& \times \exp \left[\sum_{i=1}^{q} \int_{t_{i-1}}^{t_{i}}\left[-\left(d_{2}(\tau)+\alpha_{2}(\tau) C_{0}^{*}(\tau)+\mu(\tau)\right)\right] \mathrm{d} \tau\right] \\
= & \Omega_{2} I\left(n \omega+t_{0}\right) .
\end{aligned}
$$

Therefore, for any positive integer $l_{2}$, we have $I\left(\left(n+l_{2}\right) \omega+t_{0}\right) \geq \Omega_{2}^{l_{2}} I\left(n \omega+t_{0}\right)$. It follows from (18) that

$$
I\left(\left(n+l_{2}\right) \omega+t_{0}\right) \geq \Omega_{2}^{l_{2}} I\left(n \omega+t_{0}\right) \rightarrow \infty \text {, as } l_{2} \rightarrow+\infty .
$$

From above, we obtain that $\lim I(t)=\infty$, which is a contradiction to $0<I(t)<\eta$. Thus the claim is proved, that is, there exists a $\sigma>^{t} \overrightarrow{0}^{+\infty}$ such that $\limsup _{t \rightarrow+\infty} I(t) \geq \sigma$.

Therefore, the claim is proved.

By the claim, we are left to consider the following two possibilities:

Case 1. $I(t)>\sigma$ for $t$ large enough;

Case 2. $I(t)$ oscillates about $\sigma$ for $t$ large enough.

Define $\phi=\min \left\{\frac{\sigma}{2}, \phi^{*}\right\}$ and $\phi^{*} \triangleq \sigma \exp \left[-\omega\left(d_{2}^{M}+\alpha_{2}^{M} C_{0}^{* M}+\mu^{M}\right)\right]$. We hope to show that $I(t) \geq \phi$ for $t$ large enough. The conclusion is evident in the first case. For the second case, let $t^{*}>0$ and $\xi>0$ satisfy

$$
I\left(t^{*}\right)=I\left(t^{*}+\xi\right)=\sigma
$$

and $I(t)<\sigma$ for $t^{*}<t<t^{*}+\xi$, where $t^{*}$ is large enough, such that

$$
S(t) \geq \frac{\Lambda^{L}}{\beta^{*} \eta+d_{1}^{M}+\alpha_{1}^{M} C_{0}^{* M}}-\sigma,
$$

for $t^{*}<t<t^{*}+\xi$. I $(t)$ is uniformly continuous. Hence, there is a $\lambda \quad(0<\lambda<\omega$, and $\lambda$ is independent of 
the choice of $\left.t^{*}\right)$ such that $I(t)>\frac{\sigma}{2}$ for $t^{*}<t<t^{*}+\lambda$. If $\xi<\lambda$, there is nothing to prove. Let us consider the case $\lambda<\xi$, there are two possible cases for $\xi$.

(1) If $\lambda<\xi \leq \omega$, then from system (7), we have

$$
\begin{aligned}
\frac{\mathrm{d} I(t)}{\mathrm{d} t} & =f_{i}(t, S, I)-d_{2}(t) I(t)-\alpha_{2}(t) C_{0}(t) I(t)-\mu(t) I(t) \\
& \geq\left[-\left(d_{2}^{M}+\alpha_{2}^{M} C_{0}^{* M}+\mu^{M}\right)\right] I(t) .
\end{aligned}
$$

It follows from (19) and $I\left(t^{*}\right)=\sigma$, we get

$$
\begin{aligned}
I(t) & \geq I\left(t^{*}\right) \exp \left[-\left(d_{2}^{M}+\alpha_{2}^{M} C_{0}^{* M}+\mu^{M}\right)\left(t-t^{*}\right)\right] \\
& \geq \sigma \exp \left[-\left(d_{2}^{M}+\alpha_{2}^{M} C_{0}^{* M}+\mu^{M}\right)\left(t^{*}+\lambda-t^{*}\right)\right] \\
& \geq \sigma \exp \left[-\left(d_{2}^{M}+\alpha_{2}^{M} C_{0}^{* M}+\mu^{M}\right) \omega\right] .
\end{aligned}
$$

Let $\phi^{*} \triangleq \sigma \exp \left[-\left(d_{2}^{M}+\alpha_{2}^{M} C_{0}^{* M}+\mu^{M}\right) \omega\right]$, then $I(t) \geq \phi$ for all $t \in\left[t^{*}, t^{*}+\lambda\right]$.

(2) If $\xi>\omega$, then from the discussion in subcase (1), we have $I(t) \geq \phi$ for all $t \in\left[t^{*}, t^{*}+\omega\right]$. Next, we show that $I(t) \geq \phi$ for all $t \in\left(t^{*}+\omega, t^{*}+\xi\right]$. Otherwise, there exists a constant $t^{* *}>0$ such that

$$
\begin{aligned}
& I(t) \geq \phi, \quad \text { for all } t \in\left[t^{*}, t^{*}+t^{* *}+\omega\right), \\
& I\left(t^{*}+t^{* *}+\omega\right)=\phi, \quad I(t)<\phi, \quad \text { for } 0<t-\left(t^{*}+t^{* *}+\omega\right) \ll 1 .
\end{aligned}
$$

On the other hand, similar to discussion in subcase (1), it is easy to know that we can choose a proper $\psi>0$, such that

$$
I\left(t^{*}+t^{* *}+\omega\right) \geq \psi I\left(t^{*}\right) \exp \left[-\left(d_{2}^{M}+\alpha_{2}^{M} C_{0}^{* M}+\mu^{M}\right)\left(t^{*}+t^{* *}+\omega-t^{*}\right)\right]>\phi .
$$

Since $\exp \left[-\left(d_{2}^{M}+\alpha_{2}^{M} C_{0}^{* M}+\mu^{M}\right) t\right]$ is a continuous function, that is

$$
\psi I\left(t^{*}\right) \exp \left[-\left(d_{2}^{M}+\alpha_{2}^{M} C_{0}^{* M}+\mu^{M}\right) t\right]>\phi,
$$

for $0<t \ll 1$ hold. Then for $0<t-\left(t^{*}+t^{* *}+\omega\right) \ll 1$, we have

$$
\begin{aligned}
I(t) \geq & I\left(t^{*}+t^{* *}+\omega\right) \exp \left\{-\left(d_{2}^{M}+\alpha_{2}^{M} C_{0}^{* M}+\mu^{M}\right)\left[t-\left(t^{*}+t^{* *}+\omega\right)\right]\right\} \\
\geq & \psi I\left(t^{*}\right) \exp \left[-\left(d_{2}^{M}+\alpha_{2}^{M} C_{0}^{* M}+\mu^{M}\right)\left(t^{* *}+\omega\right)\right] \\
& \times \exp \left\{-\left(d_{2}^{M}+\alpha_{2}^{M} C_{0}^{* M}+\mu^{M}\right)\left(t-t^{*}-t^{* *}-\omega\right)\right\} \\
& =\psi I\left(t^{*}\right) \exp \left[-\left(d_{2}^{M}+\alpha_{2}^{M} C_{0}^{* M}+\mu^{M}\right)\left(t-t^{*}\right)\right] \\
\geq & \phi .
\end{aligned}
$$

Then, $I(t) \geq \phi$, for $0<t-\left(t^{*}+t^{* *}+\omega\right) \ll 1$, which is a contraction. Therefore, $I(t) \geq \phi$ for $t \in\left[t^{*}, t^{*}+\xi\right]$.

Since this kind of interval $\left[t^{*}, t^{*}+\xi\right]$ is chosen in an arbitrary way (we only need $t^{*}$ to be large enough). Thus, we see that $I(t) \geq \phi$ for any $t \in\left[t^{*}, t^{*}+\xi\right]$. We conclude that $I(t) \geq \phi$ for $t$ large enough.

According to our above discussion, the choice of $\phi$ is independent of the positive solution of system (7), and we have proved that any solution of system (7) satisfies $I(t) \geq \phi$ for sufficiently large $t$, that is, $\liminf _{t \rightarrow+\infty} I(t) \geq \phi$. It is easy to obtain that, there exist positive constants $S^{*}$ such that $\liminf _{t \rightarrow+\infty} S(t) \geq S^{*}$. Therefore, system (7) is permanent. 


\section{Numerical Simulation and Conclusion}

In this paper, we have constructed an impulsive equation to model the process of periodic release of toxicant at time-varying and studied the effect of toxicant on the fish population. From a biological point of view, the most interesting results are the following. On the basis of Theorems 1 and 2, we can see that $R^{*}$ and $R_{*}$ are the threshold condition under the species and become permanent or not. Under the reasonable assumptions, we have showed that if $R^{*}<1$, the infected fish population dies out, and the disease-free periodic solution is globally asymptotically attractive. That is, if $R_{*}>1$ and the Hypotheses (A) and (B) hold, the infected fish population persists.

In the following, we will give some numerical simulations to illustrate the usefulness of the results and study the impact of impulsive release strength on the basic reproductive number. Numerical values of parameters of system (1) are given in Table 1. For the simulations that follows, we apply this set of parameters unless otherwise stated.

Table 1. Parameter values used in the numerical simulations of system (1).

\begin{tabular}{|c|c|c|}
\hline Parameter & Value & Unit \\
\hline$\Lambda(t)$ & 100 & month $^{-1}$ \\
\hline$d_{1}(t)$ & 1 & month $^{-1}$ \\
\hline$\alpha_{1}(t)$ & $1.5+0.3 \sin \frac{\pi}{6} t$ & month $^{-1}$ \\
\hline$d_{2}(t)$ & 1 & month $^{-1}$ \\
\hline$\alpha_{2}(t)$ & $2.5+0.5 \sin \frac{\pi}{6} t$ & month $^{-1}$ \\
\hline$\mu(t)$ & 2 & month $^{-1}$ \\
\hline$d_{3}(t)$ & 1 & month $^{-1}$ \\
\hline$\alpha_{3}(t)$ & $1.5+0.3 \sin \frac{\pi}{6} t$ & month $^{-1}$ \\
\hline$k(t)$ & $2+0.4 \sin \frac{\pi}{6} t$ & month $^{-1}$ \\
\hline$g(t)$ & $1.5+0.3 \sin \frac{\pi}{6} t$ & month $^{-1}$ \\
\hline$m(t)$ & $1.2+0.25 \sin \frac{\pi}{6} t$ & month $^{-1}$ \\
\hline$h(t)$ & $0.2+0.03 \sin \frac{\pi}{6} t$ & month $^{-1}$ \\
\hline$\beta_{1}(t)$ & $0.08+0.03 \sin \frac{\pi}{6} t$ & month $^{-1}$ \\
\hline$\beta_{2}(t)$ & $0.04+0.02 \sin \frac{\pi}{6} t$ & month $^{-1}$ \\
\hline$\beta_{3}(t)$ & $0.17+0.05 \sin \frac{\pi}{6} t$ & month $^{-1}$ \\
\hline$\beta_{4}(t)$ & $0.08+0.05 \sin \frac{\pi}{6} t$ & month $^{-1}$ \\
\hline$\gamma_{1}(t)$ & $0.1+0.01 \sin \frac{\pi}{6} t$ & month $^{-1}$ \\
\hline$\gamma_{2}(t)$ & $0.05+0.02 \sin \frac{\pi}{6} t$ & month $^{-1}$ \\
\hline$\gamma_{3}(t)$ & $0.1+0.01 \sin \frac{\pi}{6} t$ & month $^{-1}$ \\
\hline$\gamma_{4}(t)$ & $0.15+0.05 \sin \frac{\pi}{6} t$ & month $^{-1}$ \\
\hline$\omega$ & 12 & month \\
\hline
\end{tabular}




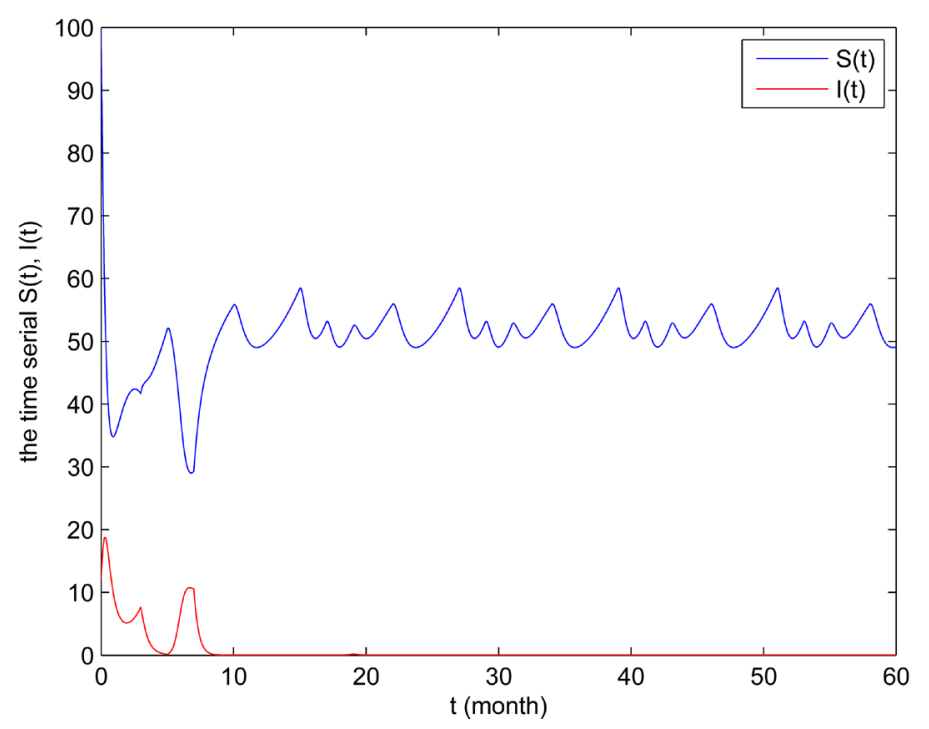

Figure 1. This figure shows that moment paths of susceptible fish $(S)$ and infected fish $(I)$ as functions of time $t . R^{*}=0.9455<1$. The infected fish will die out.

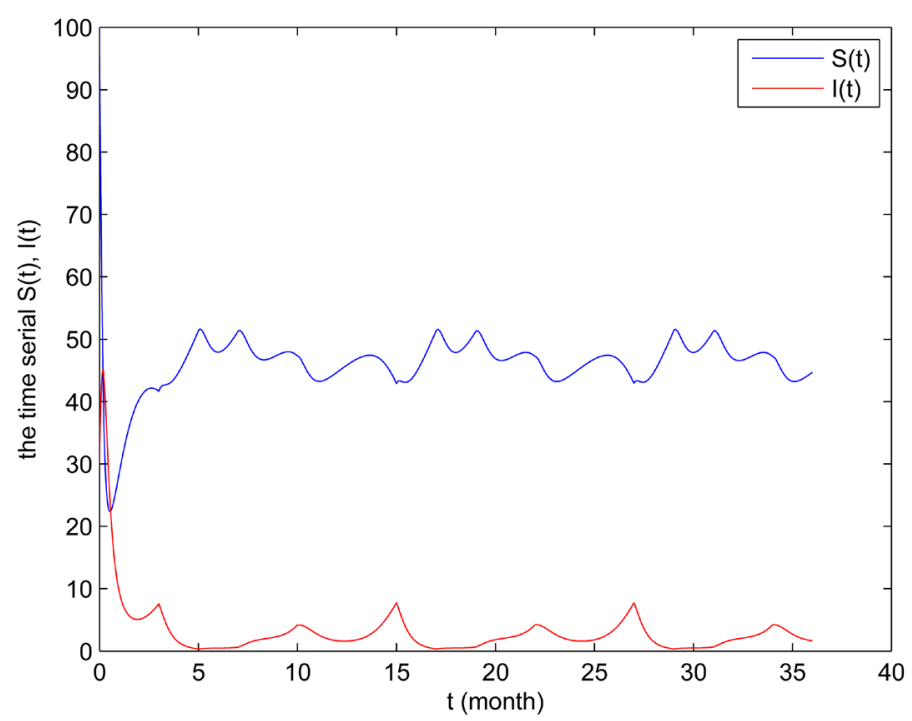

Figure 2. This figure shows that moment paths of susceptible fish $(S)$ and infected fish $(I)$ as functions of time $t . \quad R_{*}=1.1133>1$. The infected fish is uniformly persistent.

We let $q=4, t_{0}=0, t_{1}=3, t_{2}=5, t_{3}=7, t_{4}=10,\left(p_{1}, p_{2}, p_{3}, p_{4}\right)=(0.5,0.5,0.5,0.5)$ and $\left(\beta_{1}(t), \beta_{2}(t), \beta_{3}(t), \beta_{4}(t)\right)$ (see Table 1 ), then $R^{*}=0.9455$. According to Theorem 1 , we know that the disease will disappear. From Figure 1, we can also observe the disease will die out. If we choose $q=4, t_{0}=0$, $t_{1}=3, t_{2}=5, t_{3}=7, t_{4}=10,\left(p_{1}, p_{2}, p_{3}, p_{4}\right)=(0.5,0.5,0.5,0.5)$ and $\left(\gamma_{1}(t), \gamma_{2}(t), \gamma_{3}(t), \gamma_{4}(t)\right)$ (see Table 1), then $R_{*}=1.1133$. According to Theorem 2, we get that the disease will be permanent (see Figure 2). Our results cannot solve the basic reproduction number of system (8). This, of course, shows that our results have a lot of room to improve.

\section{Acknowledgements}

The research has been supported by the Natural Science Foundation of China (11261004, 11561004), the 
Natural Science Foundation of Jiangxi Province (20151BAB201016), and the Science and Technology Plan Projects of Jiangxi Provincial Education Department (GJJ14673, GJJ150984, GJJ150995). The Supporting the Development for Local Colleges and Universities Foundation of China-Applied Mathematics Innovative Team Building.

\section{References}

[1] Huang, X.P. and Gong, Y. (2007) The Research on the Current Situation and Maintainance Methods of Poyang Lake Fishing Industry. Jiangxi Fishery Sciences and Technology, 4, 1-5.

[2] Gao, S.J., Zhang, F.M. and He, Y.Y. (2013) The Effects of Migratory Bird Population in a Nonautonomous Eco-Epidemiological Model. Applied Mathematical Modelling, 37, 3903-3916. http://dx.doi.org/10.1016/j.apm.2012.07.051

[3] Freedman, H.I. and Shukla, J.B. (1991) Models for the Effect of Toxicant in Single-Species and Predator-Prey Systems. Journal of Mathematical Biology, 30, 15-30. http://dx.doi.org/10.1007/BF00168004

[4] Hallam, T.G., Clark, C.E. and Lassiter, R.R. (1983) Effects of Toxicants on Populations: A Qualitative Approach I. Equilibrium Environmental Exposure. Ecological Modelling, 8, 291-304. http://dx.doi.org/10.1016/0304-3800(83)90019-4

[5] Hallam, T.G., Clark, C.E. and Jordan, G.S. (1983) Effects of Toxicants on Populations: A Qualitative Approach II. First Order Kinetics. Journal of Mathematical Biology, 18, 25-37. http://dx.doi.org/10.1007/bf00275908

[6] Hallam, T.G. and de Luna, J.L. (1984) Effects of Toxicants on Populations: A Qualitative Approach III. Environment and Food Chain Pathways. Journal of Theoretical Biology, 109, 411-429. http://dx.doi.org/10.1016/S0022-5193(84)80090-9

[7] Hallam, T. G. and Ma, Z.E. (1986) Persistence in Population Models with Demographic Fluctuations. Journal of Mathematical Biology, 24, 327-339. http://dx.doi.org/10.1007/BF00275641

[8] Tao, F.M. and Liu, B. (2008) Dynamic Behaviors of a Single Species Population Model with Birth Pulses in a Polluted Environment. Rocky Mountain Journal of Mathematics, 38, 1663-1684. http://dx.doi.org/10.1216/RMJ-2008-38-5-1663

[9] Liu, B., Duan, Y. and Gao, Y.H. (2009) Dynamics of a Stage Structure Pest Control Model with Impulsive Effects at Different Fixed Time. Discrete Dynamics in Nature and Society, 57, 332-337. http://dx.doi.org/10.1155/2009/392852

[10] Liu, B., Duan, Y. and Shi, L. (2012) Dynamics of an SI Epidemic Model with External Effects in a Polluted Environment. Nonlinear Analysis, 13, 27-38. http://dx.doi.org/10.1016/j.nonrwa.2011.07.007

[11] Liu, B., Zhang, L. and Zhang, Q. (2009) The Effects of a Single Stage-Structured Population Model with Impulsive Toxin Input and Time Delays in a Polluted Environment. Applicable Analysis, 88, 1143-1155. http://dx.doi.org/10.1080/00036810903156156

[12] Liu, H.P. and Ma, Z.E. (1991) The Threshold of Survival for the System of Two Species in a Polluted Environment. Journal of Mathematical Biology, 30, 49-61. http://dx.doi.org/10.1007/BF00168006

[13] Ma, Z., Cui, G. and Wang, W. (1990) Persistence and Extinction of a Population in a Polluted Environment. Mathematical Biosciences, 101, 75-97. http://dx.doi.org/10.1016/0025-5564(90)90103-6

[14] Ma, Z.E., Song, B.J. and Hallam, T.G. (1989) The Threshold of Survival for the System in Fluctuating Environment. Bulletin of Mathematical Biology, 51, 311-323. http://dx.doi.org/10.1007/BF02460110

[15] Debasis, M. (2002) Persistence and Global Stability of a Population in a Polluted Environment with delay. Journal of Biological Systems, 10, 225-232. http://dx.doi.org/10.1142/S021833900200055X

[16] Pal, A.K. and Samanta, G.P. (2010) A Single Species Population in a Polluted Environment. International Journal of Biomathematics, 3, 187-204. http://dx.doi.org/10.1142/S1793524510000933

[17] Zhao, Z., Chen, L.S. and Song, X.Y. (2009) Extinction and Permanence of Chemostat Model with Pulsed Input in a Polluted Environment. Communications in Nonlinear Science and Numerical Simulation, 14, 1737-1745. http://dx.doi.org/10.1016/j.cnsns.2008.01.009

[18] Wang, F. and Ma, Z.E. (2004) Persistence and Periodic Orbits for an SIS Model in a Polluted Environment. Computers \& Mathematics with Applications, 47, 779-792. http://dx.doi.org/10.1016/S0898-1221(04)90064-8

[19] Liu, B., Chen, L.S. and Zhang, Y.J. (2003) The Effects of Impulsive Toxicant Input on a Population in a Polluted Environment. Journal of Biological Systems, 11, 265-274. http://dx.doi.org/10.1142/S0218339003000907

[20] Zhang, T.L. and Teng, Z.D. (2007) On a Nonautonomous SEIRS Model in Epidemiology. Bulletin of Mathematical Biology, 69, 2537-2559. http://dx.doi.org/10.1007/s11538-007-9231-z

[21] He, Y.Y., Gao, S.J. and Xie, D.H. (2013) An SIR Epidemic Model with Time-Varying Pulse Control Schemes and Sa- 
turated Infectious Force. Applied Mathematical Modelling, 37, 8131-8140. http://dx.doi.org/10.1016/j.apm.2013.03.035

[22] Liu, W.M., Levin, S.A. and Iwasa, Y. (1986) Inuence of Nonlinear Incidence Rates upon the Behavior of SIRS Epidemiological Models. Journal of Mathematical Biology, 23, 187-204. http://dx.doi.org/10.1007/BF00276956

[23] Hu, Z.X., Bi, P., Ma, W.B. and Ruan, S.G. (2011) Bifurcations of an SIRS Epidemic Models with Nonlinear Incidence Rate. Discrete. Discrete and Continuous Dynamical Systems—Series B, 15, 93-112. http://dx.doi.org/10.3934/dcdsb.2011.15.93

[24] Huang, G., Ma, W.B. and Takeuchim, Y. (2011) Global Analysis for Delay Virus Dynamics Model with Beddington-DeAngelis Functional Response. Applied Mathematics Letters, 24, 1199-1203. http://dx.doi.org/10.1016/j.aml.2011.02.007

[25] D’Onofrio, A. (2005) Vaccination Policies and Nonlinear Force of Infection: Generalization of an Observation by Alexander and Moghadas. Applied Mathematics and Computation, 168, 613-622. http://dx.doi.org/10.1016/j.amc.2004.09.013

[26] He, Y.Y., Gao, S.J., Lv, H.M. and Liu, Y.J. (2013) Asymptotic Behavior of an SEIR Epidemic Model with Quadratic Treatment. Journal of Applied Mathematics and Computing, 42, 245-257. http://dx.doi.org/10.1007/s12190-012-0617-1

[27] Zhang, X.Y., Gao, S.J. and Cao, H.H. (2014) Threshold Dynamics for a Nonautonomous Schistosomiasis Model in a Periodic Environment. Journal of Applied Mathematics and Computing, 46, 305-319. http://dx.doi.org/10.1007/s12190-013-0750-5

[28] Zhang, Y., Gao, S.J., Fan, K.G. and Wang, Q.Y. (2014) Asymptotic Behavior of a Nonautonomous Predator-Prey Model with Hassell-Varley Type Functional Response and Random Perturbation. Journal of Applied Mathematics and Computing, 49, 573-594. http://dx.doi.org/10.1007/s12190-014-0854-6

[29] Zhang, F.M., Gao, S.J. and Zhang, Y. (2012) The Effects of Pulse Culling on Population Growth of Migratory Birds and Economical Birds. Nonlinear Dynamics, 67, 767-779. http://dx.doi.org/10.1007/s11071-011-0025-3 